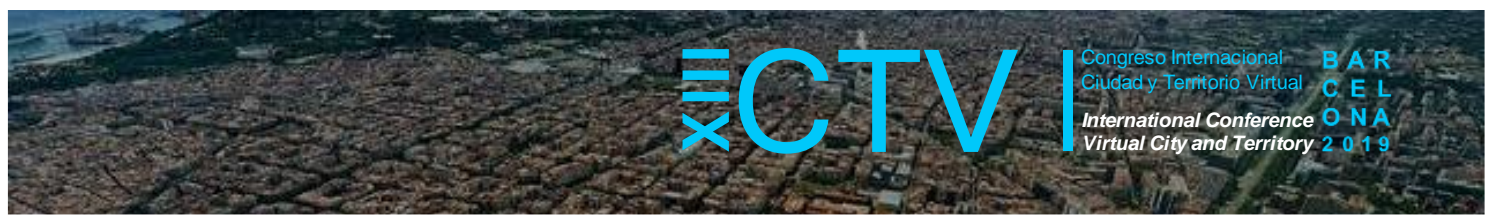

\title{
EL DERECHO A LA CIUDAD DESDE LA EXCLUSIÓN RESIDENCIAL: LA EVOLUCIÓN DE LOS BARRIOS VULNERABLES DE LA COMUNITAT VALENCIANA
}

\author{
Caravantes López de Lerma, Glòria Maria ${ }^{1 *}$
}

Remisión inicial: 2019-06-16; Remisión definitiva: 2019-09-08; Publicación: 2019-12-21

Citación: Caravantes, G. M. (2019). El Derecho a la Ciudad desde la exclusión residencial: La evolución de los barrios vulnerables de la Comunitat Valenciana. En XIII CTV 2019 Proceedings: XIII International Conference on Virtual City and Territory: "Challenges and paradigms of the contemporary city": UPC, Barcelona, October 2-4, 2019. Barcelona: CPSV, 2019, p. 8661. E-ISSN 2604-6512. DOI http://dx.doi.org/10.5821/ctv.8661

\section{Resumen}

El derecho a la ciudad fue un concepto empleado por primera vez por Lefebvre (1968) entendido como aquel derecho de la ciudadanía a constituir ciudades que respondan a las necesidades humanas. De este modo, las necesidades de una ciudad no son más que la manifestación expresa de las necesidades colectivas de sus residentes reflejadas en el espacio urbano y comunitario (Caravantes, 2018). A las necesidades colectivas cabe sumársele las necesidades específicas de las personas que forman parte activa e integrante de las ciudades. La concentración de tales necesidades, así como de fenómenos vinculados al ámbito residencial conllevan a la "acumulación de clases desfavorecidas en las ciudades" (Subirats, 2016, p. 43). Por su parte, Alguacil et al. (2014, p. 77) refieren que esta acumulación hace referencia a la vulnerabilidad urbana, entendida como "aquella potencialidad de que la población de un determinado espacio urbano concreto sea afectada por alguna(s) circunstancia(s) adversa(s). La desigualdad social y la fragilidad que se genera en la estructuración de determinados territorios y la ubicación de grupos sociales en los mismos (Subirats, 2005), se explica a través de dos procesos diferentes: el primero de ellos manifestado a través del perfil socio-demográfico y socio-económico de la comunidad, donde confluyen etnias minoritarias. En segundo lugar, por las características estructurales de los barrios, incluidas en éstas tanto la disponibilidad de los recursos y/o servicios como el acceso a los mismos. En este último punto, el alejamiento de los recursos comunitarios determina el nivel de bienestar disponible para la población y sus carencias, marcarán sus puntos más sensibles para la comunidad. En base a ello, existen diferentes instrumentos a nivel estatal y autonómico que permiten identificar geográficamente la ubicación de entornos vulnerables en las ciudades. Desde el Atlas de la Vulnerabilidad Urbana del Ministerio de Fomento, pasando por estudios autonómicos como el Mapa de Riesgo Social de Zaragoza (MRSZ), la identificación de ámbitos de vulnerabilidad residencial de Barcelona, al Visor de Espacios Urbanos Sensibles (VEUS) de la Comunitat Valenciana desarrollado por la Conselleria de Vivienda, Obras Públicas y Urbanismo. En este último caso, el VEUS mide la vulnerabilidad urbana a partir de secciones censales, en base a variables de carácter residencial, socioeconómico y socio-demográficas. A partir de ello, se identifican tres tipologías de vulnerabilidad urbana concernientes a: vulnerabilidad integral, polivulnerabilidad y vulnerabilidad residual. Con ello, el objeto de la presente comunicación se corresponde con analizar la evolución de los barrios vulnerables de la CV desde $1991-2016$. No obstante, las fuentes que permiten realizar un estudio de panel en base a ese periodo, se identifican con el Atlas de la Vulnerabilidad Urbana y el Mapa sobre Vivienda y Comunidad Gitana en España. Además de ello, la identificación territorial por tipologías de vulnerabilidad desarrollada por el VEUS, permite además cartografiar cada una de las provincias por sección censal e identificar los núcleos territoriales con mayores índices de vulnerabilidad residencial, socioeconómica y socio-demográfica. Según los resultados obtenidos a través del Ministerio, la CV ha experimentado un incremento de barrios vulnerables similar al acontecido en España. En 1991 se delimitaron un total de 24 barrios vulnerables en 9 ciudades, mientras que en 2001 se identificaron 38 en 12 ciudades. Las dinámicas de concentración poblacional responden a "una serie de inercias que forman parte del sistema en que vivimos, que se encuentran presentes tanto en las políticas que conducen a la pobreza como, en ocasiones, en las fuerzas que intentan combatirlas" (Varea et al., 2016, p. 100). Según el último informe FOESSA (2019), las personas migrantes y de etnia gitana se encuentran entre aquellos perfiles con mayor riesgo de exclusión social, duplicando la tasa de exclusión social de la CV con respecto al resto de CC.AA. De forma paralela, si contemplamos los datos de la Fundación Secretariado Gitano, València es la provincia valenciana donde mayor índice de barrios o asentamientos con población gitana dispone (5.534), seguida de Alicante con 5.515 (Ministerio de Sanidad, Servicios Sociales e Igualdad, 2016). De

1 Trabajadora social y Máster en Cooperación Internacional al Desarrollo, Universitat de Valencia (UPV), https://orcid.org/0000-0003-2656-7032. * Correo de contacto: gloriamariacaravantes@gmail.com 


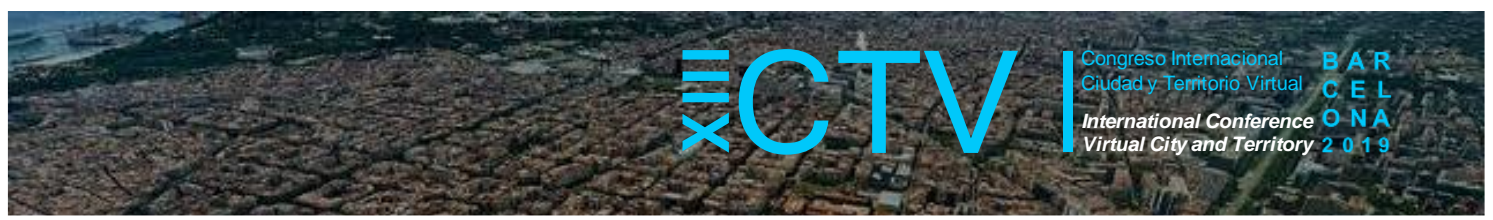

este modo, entre los resultados se aportan, tanto datos cuantitativos que evidencian la emergencia, cada vez más notable de barrios vulnerables, como mapas de la vulnerabilidad urbana de la Comunitat Valenciana donde aparecen reflejados este tipo de entornos en base a las tres provincias. Asimismo, el VEUS (2018) identificó un total de 830 secciones censales con una representación de los Espacios Urbanos Sensibles (EUS) del $24 \%$ siendo en Alicante un $37,47 \%$ (458), València con 17,92\% (324) y Castellón con un 10,48\% (48). En este sentido, la identificación de EUS abarca un mayor espacio territorial que la delimitación administrativa ofrecida por el Atlas de la Vulnerabilidad y ofreciendo una radiografía de la CV más detallada desde el ámbito local y de proximidad. Entre las principales conclusiones cabe destacar que, tanto en la configuración del derecho a la ciudad como en la identificación de aquellos espacios marcados por la vulnerabilidad, el fenómeno de la exclusión residencial es una cuestión esencial que no puede eludirse desde los poderes públicos en la garantía de este derecho. El abandono de las políticas públicas en pro del derecho a la ciudad, agrava y cronifica las desigualdades existentes en este tipo de entornos urbanos. Actualmente, el argumentario político continúa escudándose en la ya consolidada crisis económica y encontrando importantes resistencias a desarrollar una gobernanza territorial de carácter local que permita atajar los procesos de desigualdad.

\section{Abstract}

The right to the city was a concept used for the first time by Lefebvre (1968) understood as that right of citizenship to constitute cities that respond to human needs. In this way, the needs of a city are nothing more than the express manifestation of the collective needs of its residents reflected in the urban and community space (Caravantes, 2018). The specific needs of the people who are an active and integral part of the cities can be added to the collective needs. The concentration of such needs, as well as phenomena linked to the residential environment, lead to the "accumulation of disadvantaged classes in cities" (Subirats, 2016, p. 43). On the other hand, Alguacil et al. (2014, p. 77 ) report that this accumulation refers to urban vulnerability, understood as "that potentiality of the population of a specific urban space being affected by some adverse circumstance (s). The social inequality and fragility that is generated in the structuring of certain territories and the location of social groups in them (Subirats, 2005), is explained through two different processes: the first one manifested through the socio-social profile. demographic and socio-economic community, where minority ethnic groups converge. Secondly, due to the structural characteristics of the neighborhoods, including the availability of resources and / or services and access to them. In this last point, the distancing of community resources determines the level of well-being available to the population and their deficiencies, they will mark their most sensitive points for the community. Based on this, there are different instruments at state and regional level that allow geographically identifying the location of vulnerable environments in cities. From the Atlas of Urban Vulnerability of the Ministry of Development, through autonomous studies such as the Social Risk Map of Zaragoza (MRSZ), the identification of areas of residential vulnerability in Barcelona, to the Viewer of Sensitive Urban Spaces (VEUS) of the Community Valenciana developed by the Department of Housing, Public Works and Urban Planning. In the latter case, VEUS measures urban vulnerability based on census sections, based on residential, socioeconomic and sociodemographic variables. From this, three types of urban vulnerability are identified concerning: integral vulnerability, polyvulnerability and residual vulnerability. With this, the purpose of this communication corresponds to analyzing the evolution of the vulnerable neighborhoods of the CV since 1991 - 2016. However, the sources that allow to carry out a panel study based on that period, are identified with the Atlas of Urban Vulnerability and the Map on Housing and Roma Community in Spain. In addition to this, the territorial identification by types of vulnerability developed by the VEUS, also allows mapping each of the provinces by census section and identifying the territorial nuclei with the highest rates of residential, socioeconomic and socio-demographic vulnerability. According to the results obtained through the Ministry, the CV has experienced an increase in vulnerable neighborhoods similar to what happened in Spain. In 1991, a total of 24 vulnerable neighborhoods were defined in 9 cities, while in 200138 were identified in 12 cities. The dynamics of population concentration respond to "a series of inertia that are part of the system in which we live, which are present both in the policies that lead to poverty and, sometimes, in the forces that try to combat them" (Varea et al., 2016, p. 100). According to the latest FOESSA report (2019), migrant and Roma people are among those profiles with the highest risk of social exclusion, doubling the rate of social exclusion of the CV with respect to the rest of Autonomous Communities. If we look at the data of the Fundación Secretariado Gitano, Valencia is the Valencian province where the highest index of neighborhoods or settlements with Roma population has $(5,534)$, followed by Alicante with 5,515 (Ministry of Health, Social Services and Equality, 2016). In this way, the results provide both quantitative data that show the emergence, increasingly noticeable of vulnerable neighborhoods, and maps of the urban vulnerability of the Valencian Community where these types of environments are reflected based on the three provinces. Likewise, VEUS (2018) identified a total of 830 census sections with a representation of Sensitive Urban Spaces (EUS) of 24\%, being in Alicante $37.47 \%$ (458), Valencia with $17.92 \%$ (324) and Castellón with $10.48 \%$ (48). In this sense, the identification of EUS encompasses a larger territorial space than the administrative delimitation offered by the Vulnerability Atlas and offering a more detailed X-ray of the CV from the local and proximity level. Among the main conclusions, it should be noted that, both in the configuration of the right to the city and in the identification of those spaces marked by vulnerability, the phenomenon of residential exclusion is an essential issue that cannot be avoided by the public authorities in the guarantee of this right. The abandonment of public policies in favor of the right to the city aggravates 


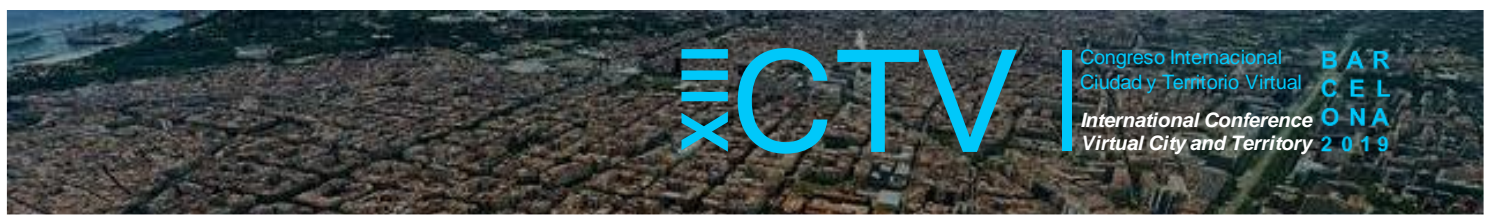

and chronicles the inequalities existing in this type of urban environment. Currently, the political argument continues to be scrutinized in the already consolidated economic crisis and encountering significant resistance to developing a territorial governance of a local nature that allows to tackle the processes of inequality.

Palabras Clave: Derecho a la ciudad; barrios vulnerables; Comunidad Valenciana; vulnerabilidad urbana

Key words: Right to the city; vulnerable neighborhoods; Valencian region; urban vulnerability

\section{Introducción}

En los últimos años, las desigualdades entre regiones, ciudades y territorios han ido incrementándose progresivamente, derivando en un aumento paulatino de la vulnerabilidad y la exclusión social. De acuerdo con Naciones Unidas (2018, pág. 3): "detener y corregir el aumento de la desigualdad también es vital para que en adelante el crecimiento sea equilibrado y sostenible". En el ámbito urbano, ONU - Hábitat ha subrayado el aumento de los asentamientos precarios en las ciudades y, en consecuencia, se han desarrollado actuaciones institucionales tales como el Programa Participativo de Mejoramiento de Barrios Precarios cuya finalidad principal es la mejora de la situación de las viviendas y las condiciones de vida de tales asentamientos en la actualidad. La variable residencial en el ámbito urbano constituye un elemento esencial en la configuración de las ciudades y en la calidad de vida de sus residentes. En este sentido, la dimensión ambiental y residencial de las ciudades es el resultado visible de una concatenación del propio proceso de exclusión social. Del mismo modo que la exclusión social contempla múltiples causas y dimensiones, en la exclusión residencial adquiere especial relevancia el espacio urbano. El poder del mercado inmobiliario en España, ha generado en los últimos años una crisis de accesibilidad, es decir, "a la exclusión del acceso a la vivienda se añade la persistencia y constante emergencia de barrios degradados, viviendas inadecuadas y habitabilidad sin condiciones" (Subirats, et al., 2004, p. 28). Asimismo, desde la enunciación de los Objetivos de Desarrollo Sostenible, especialmente en el décimo primero sobre Ciudades y Comunidades Sostenibles se destaca que: "el futuro que queremos incluye a ciudades de oportunidades, con acceso a servicios básicos, energía, vivienda, transporte y más facilidades para todos". En las metas del propio ODS 11 "converge indirectamente el desiderátum de gozar de un conjunto de derechos y garantías relativas a la calidad de vida de la ciudadanía" (Caravantes, 2018, pág.104), tal y como queda reflejado en la Tabla 1.

Tabla 1. Resumen de las metas establecidas para el Objetivo de Desarrollo Sostenible 11

\begin{tabular}{ll}
$\begin{array}{l}\text { 1. Vivienda y barrios } \\
\text { marginales }\end{array}$ & $\begin{array}{l}\text { Asegurar el acceso a la vivienda y servicios básicos } \\
\text { adecuados, seguros y asequibles, y mejorar las zonas } \\
\text { marginales. }\end{array}$ \\
\hline $\begin{array}{l}\text { 2. Transporte sostenible } \\
\text { 3.Planificación }\end{array}$ & $\begin{array}{l}\text { Proporcionar acceso a sistemas de transporte seguros, } \\
\text { públicos, asequibles, accesibles y sostenibles y mejorar } \\
\text { la seguridad vial, prestando especial atención a las } \\
\text { necesidades de las personas en situación vulnerable. }\end{array}$ \\
\hline & $\begin{array}{l}\text { Aumentar la urbanización inclusiva y sostenible y la } \\
\text { capacidad para una planificación y gestión participativas, } \\
\text { integradas y sostenibles de los asentamientos humanos } \\
\text { en todos los países. }\end{array}$ \\
\hline
\end{tabular}




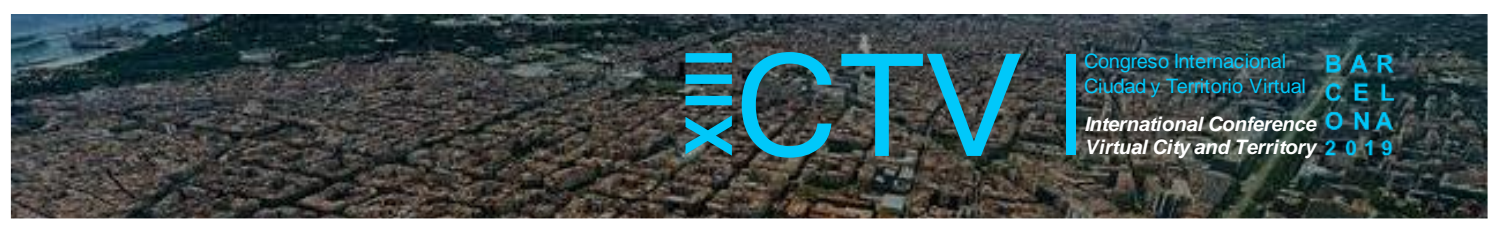

\begin{tabular}{|c|c|c|}
\hline $\int_{001}^{4}$ & $\begin{array}{l}\text { 4.Patrimonio cultural y } \\
\text { natural }\end{array}$ & $\begin{array}{l}\text { Redoblar los esfuerzos para proteger y salvaguardar el } \\
\text { patrimonio cultural y natural del mundo. }\end{array}$ \\
\hline & $\begin{array}{l}\text { 5. Reducción de riesgos de } \\
\text { desastres }\end{array}$ & $\begin{array}{l}\text { Reducir las muertes y las personas afectadas por los } \\
\text { desastres, y las pérdidas económicas derivadas, con } \\
\text { especial atención a las personas en situación vulnerable. }\end{array}$ \\
\hline & $\begin{array}{l}\text { 6. Calidad del aire y } \\
\text { manejo de residuos }\end{array}$ & $\begin{array}{l}\text { Reducir el impacto ambiental de las ciudades, con } \\
\text { especial atención a la calidad del aire y a la gestión de } \\
\text { los residuos. }\end{array}$ \\
\hline & 7. Espacios públicos & $\begin{array}{l}\text { Proporcionar zonas verdes y espacios públicos, } \\
\text { seguros, inclusivos y accesibles, con especial atención } \\
\text { a colectivos en situación vulnerable. }\end{array}$ \\
\hline & $\begin{array}{l}\text { 8. Vinculación urbano-rural } \\
\text { y planificación regional }\end{array}$ & $\begin{array}{l}\text { Fortalecer los vínculos económicos sociales y } \\
\text { ambientales positivos entre las zonas urbanas, } \\
\text { periurbanas y rurales por medio de la planificación al } \\
\text { desarrollo nacional y regional. }\end{array}$ \\
\hline & $\begin{array}{l}\text { 9. Cambio climático y } \\
\text { resiliencia }\end{array}$ & $\begin{array}{l}\text { Aumentar el número de ciudades que adopten y pongan } \\
\text { en marcha políticas y planes para promover la inclusión, } \\
\text { el uso efectivo de recursos, combatir el cambio climático } \\
\text { y gestionar los riesgos de desastres de todos los niveles. }\end{array}$ \\
\hline-5 & 10. Edificios sostenibles & $\begin{array}{l}\text { Proporcionar apoyo a los países menos adelantados } \\
\text { para que puedan construir edificios sostenibles y } \\
\text { resilientes con materiales locales. }\end{array}$ \\
\hline
\end{tabular}

Fuente: ONU HABITAT (2015).

En la misma línea que Naciones Unidas, Lefebvre (1969) acuñó el concepto del derecho a la ciudad en cuyas palabras afirma que: "el derecho a la ciudad no puede concebirse como un simple derecho de visita o retorno hacia las ciudades tradicionales. Sólo puede formularse como derecho a la vida urbana, transformada, renovada (Lefebvre, 1969 pág. 138). Su teoría no finaliza únicamente en el planteamiento del derecho a la ciudad, sino que asimismo realiza dos proposiciones para desarrollar una estrategia urbana que necesariamente, ha de estar sustentada tanto en apoyo social como en las fuerzas políticas para materializarse:

a) Un programa político de reforma urbana: Lefebvre aboga por un programa político fundado en la singularidad y especificidad. El mismo, ha de ser acometido por los partidos políticos, especialmente de aquellos que representen los intereses de la clase obrera.

b) Proyectos urbanísticos: Para ello, manifiesta que tales proyectos incluyan "«modelos», formas de espacio y tiempos urbanos, sin preocuparse de su carácter realizable actualmente o no, utópico o no (es decir, proyectos lúcidamente "utópicos»)" (Lefebvre, 1969 , p. 134). En definitiva, proyectos urbanísticos al servicio del estilo de vida de las personas y al desarrollo de lo urbano. 


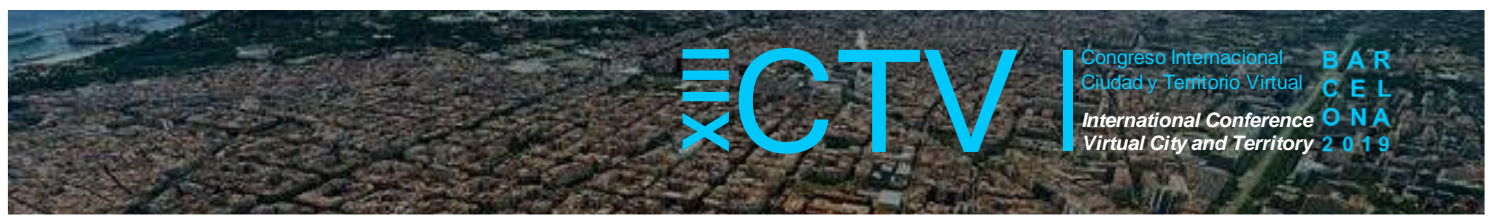

Ante tales proposiciones, el propio autor manifiesta que en la actualidad la afirmación de este derecho puede llegar a resultar utópica. Por todo ello, el planteamiento lefebvriano del siglo XIX sigue aún vigente permitiendo profundizar en el estudio del derecho a la ciudad, siendo reconocida su teoría en agencias supranacionales y agendas políticas, entre otras (Busquet y Garnier, 2011). La adopción de su teoría va más allá de vislumbrar la ciudad desde la perspectiva tradicional-industrial abriéndose paso a una nueva concepción de una ciudad global. Sin embargo, declara que el coste de no reconocer el derecho a la ciudad, es más elevado que llevarlo a la práctica. En similitud con dicha cuestión, Jane Jacobs refirió que "si en una vecindad se pueden introducir las condiciones que generan diversidad y si se estimulan en lugar de obstaculizarse los indicios de rehabilitación, creo que ningún barrio bajo tiene por qué ser perpetuo" (Jacobs, 2011, pág. 309). Ante dicha cuestión, en los sucesivos epígrafes se describe el estudio realizado en la Comunitat Valenciana respecto a la evolución de los barrios y espacios vulnerables en el periodo 1991 - 2016 en las provincias de València, Castellón y Alicante a partir de distintas herramientas y plataformas tales como el Atlas de la Vulnerabilidad Urbana (1991; 2006; 2011), el Mapa sobre Vivienda y Comunidad Gitana en España (2015; 2018) y el nuevo Visor de Espacios Urbanos Sensibles (VEUS) desarrollado por la Conselleria de Vivienda, Obras Públicas y Urbanismo.

\subsection{El punto de partida: análisis y contextualización de la Comunitat Valenciana}

En los últimos años y a consecuencia de la crisis económica, la situación social y económica de la sociedad valenciana ha ido progresivamente derivando en el surgimiento de nuevas necesidades (desahucios, búsqueda de asilo y refugio, contaminación medioambiental...). El aumento de las situaciones de riesgo y de empobrecimiento ha afectado en la actualidad a sectores cada vez más amplios de nuestra sociedad, obligando a los poderes públicos a ofrecer respuestas capaces de adaptarse a las nuevas realidades sociales. En el caso que nos ocupa, la tasa de riesgo de pobreza según la media española sitúa a la Comunitat Valenciana en las últimas posiciones junto con la Región de Murcia, Castilla-La Mancha, Andalucía, Canarias, Extremadura y Ceuta.

\section{Gráfico 1. Mapa de la tasa de riesgo de pobreza según el umbral español por CCAA \%}
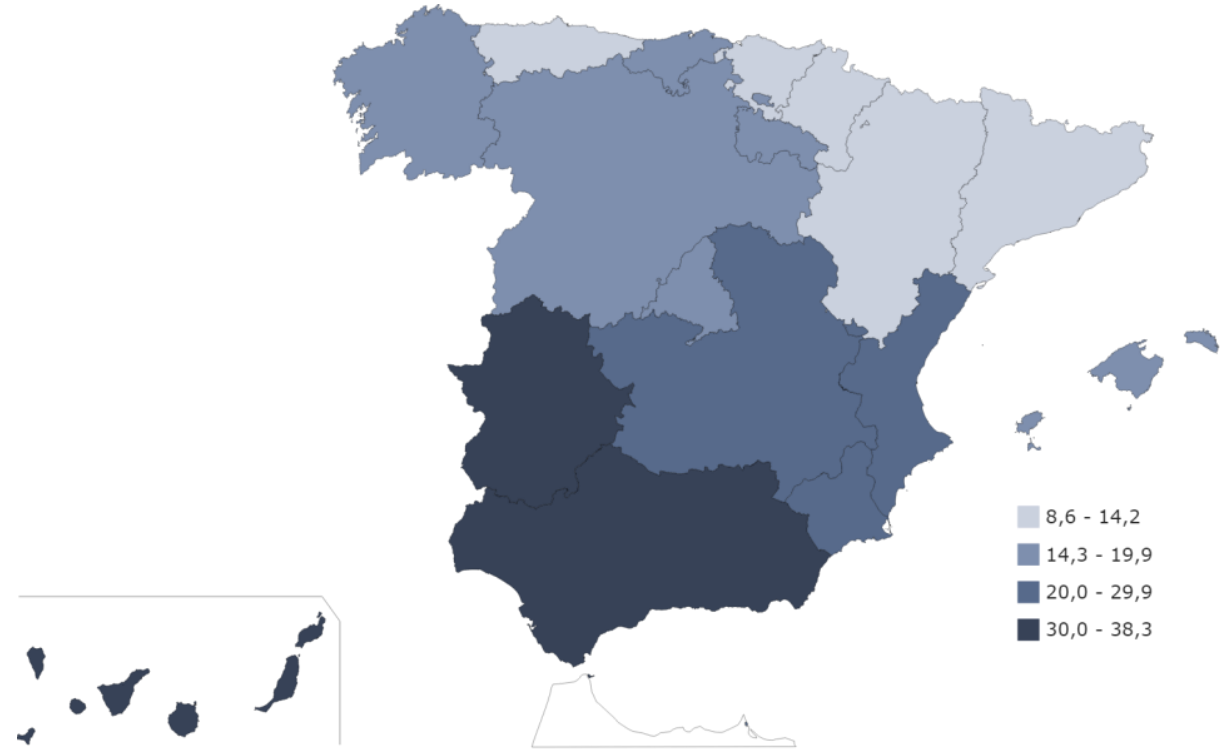

Fuente: INE (2018). 


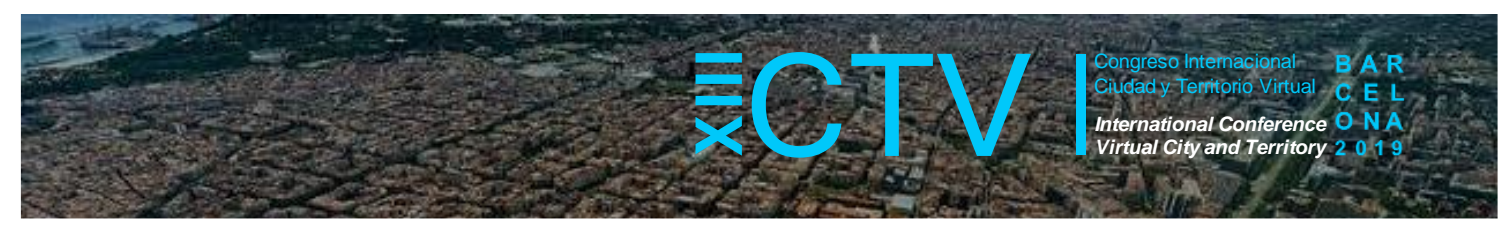

Considerando algunos datos de esta cuestión, la tasa AROPE (2017) destaca que el riesgo de pobreza y/o exclusión social en la Comunitat se sitúa en 32,9\% del total poblacional, es decir, un total de 1.638.617 personas en situación de riesgo. Según la última Encuesta de Vida (ECV, 2019) cerca del $22,8 \%$ de la población residente en la Comunitat Valenciana está por debajo del umbral de riesgo de pobreza según la distribución de la renta en 2017 y un 27,6\% se encuentra en riesgo de pobreza o exclusión social (1,8 inferior a los datos de la ECV de 2017). Además de ello, resulta característico que cerca del $70 \%$ (0,6 puntos menos que el año anterior) de los hogares en la Comunitat Valenciana perciben prestaciones sociales (dependencia, renta valenciana de inclusión, ayudas de emergencia, prestación por desempleo...). Ante esta cuestión, la concentración demográfica de quienes comparten características similares (bajos niveles de renta, deterioro y degradación de las unidades residenciales, precariedad laboral...) subraya la variable territorial del fenómeno de la vulnerabilidad destacando que: "la dimensión del barrio se erige como un espacio particularmente significativo para comprender la dialéctica inclusión/exclusión social urbana, y proporciona información substancial sobre los factores que la determinan y las prácticas y estrategias que las personas ponen en marcha respecto a ellos" (FOESSA, 2008, pág. 285286). Asimismo, los últimos datos disponibles del Informe FOESSA (2014) sobre la Comunitat Valenciana ${ }^{2}$ señalan que los perfiles con mayor riesgo de exclusión social se identifican con las personas migrantes y de etnia gitana "duplicando, en este sentido, la tasa de exclusión social de la población de la Comunitat Valenciana (...) el riesgo de exclusión social para ambos colectivos, es muy superior a la media española, situado en un $73 \%$ para la población inmigrante y un $84 \%$ para las personas de etnia gitana, siendo la media nacional $52,5 \%$ y $72 \%$ respectivamente (EINSFOESSA, 2013)" (cit. en Uceda-Maza, et al., 2018, pág. 357).

\section{Evolución de los barrios vulnerables en la Comunitat Valenciana 1991-2016}

Con la finalidad de ahondar en el objeto de la presente ponencia "analizar la evolución de los barrios vulnerables de la Comunitat Valenciana desde 1991 - 2016", se partirá de tres fuentes estadísticas (dos nacionales y una autonómica) que permiten abordar dicha cuestión.

Tomando como punto de partida el Atlas de Barrios Vulnerables de España ${ }^{3}$ (2015) y el Atlas de la Vulnerabilidad Urbana ${ }^{4}$ del Ministerio de Fomento, para el periodo 1991 - 2001 la vulnerabilidad urbana en España se incrementó hasta un 60\%, teniendo su traslación en el ámbito territorial en la existencia de 378 barrios vulnerables para el primer periodo y 604 para el año 2001 (con un crecimiento demográfico de un 4\% para el mismo periodo).

En lo que respecta a la Comunitat Valenciana, también se experimentó un crecimiento similar en la identificación de barrios vulnerables, siendo 24 barrios vulnerables en 1991 y 38 barrios

\footnotetext{
2 Recientemente ha sido publicado el VIII Informe FOESSA. La exclusión social se enquista en una sociedad cada vez más desvinculada. No obstante, hasta el último trimestre de 2019 no estarán disponible los datos específicos sobre la Comunitat Valenciana.

3 Basado en los datos recogidos en el "Análisis urbanístico de Barrios Vulnerables" de 1991 y 2001 y su adenda en 2006. El Atlas de Barrios Vulnerables de España ofrece un análisis estadístico y cartográfico de los mencionados barrios en las ciudades de más de 50.000 habitantes.

${ }^{4}$ Aplicación web dispone de información estadística y desarrollar mapas sobre vulnerabilidad urbana en España a partir de los censos de Población y Viviendas de 2001 y de 2011. Asimismo, se organiza entorno a 4 dominios: 1) Indicadores de Vulnerabilidad Urbana; 2) Análisis contextual de la Vulnerabilidad Urbana; 3) Índices de Desigualdad Urbana y 4) Índices Sintéticos de Vulnerabilidad Urbana.
} 


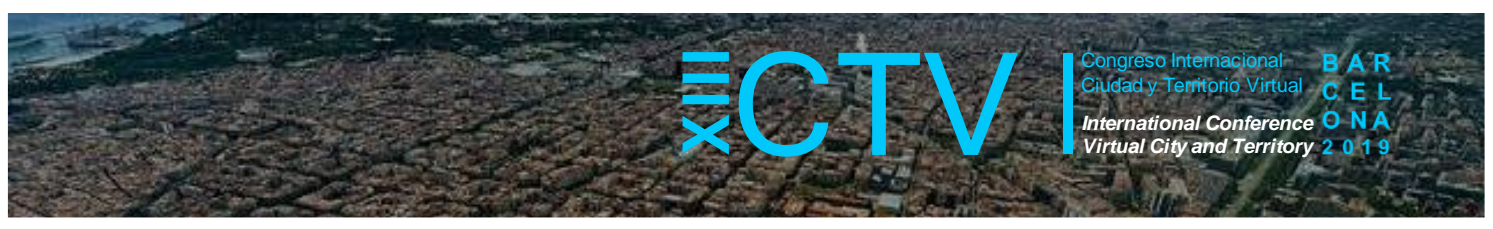

en el año 20115. En la adenda del Atlas de Barrios Vulnerables de 2006 (2015), puede apreciarse cómo dichas cifras se han mantenido estables, aunque con un aumento en la población vulnerable residente en este tipo de entornos tal y como se refleja en la Tabla 2:

Tabla 2. Evolución de Barrios Vulnerables en la Comunitat Valenciana 1991-2006 ${ }^{6}$

\begin{tabular}{|c|c|c|c|c|c|c|c|c|c|c|c|c|}
\hline & \multicolumn{4}{|c|}{1991} & \multicolumn{4}{|c|}{2001} & \multicolumn{4}{|c|}{2006} \\
\hline & $\begin{array}{l}\text { POB. } \\
1991\end{array}$ & $\begin{array}{c}\text { № } \\
\text { BBVV }\end{array}$ & $\begin{array}{l}\text { POB. } \\
\text { Vuln. }\end{array}$ & $\begin{array}{l}\% \text { POB. } \\
\text { Vuln. }\end{array}$ & $\begin{array}{l}\text { POB. } \\
2001\end{array}$ & $\begin{array}{c}\text { № } \\
\text { BBVV }\end{array}$ & $\begin{array}{l}\text { POB. } \\
\text { Vuln. }\end{array}$ & $\begin{array}{l}\% \text { POB. } \\
\text { Vuln. }\end{array}$ & $\begin{array}{l}\text { POB. } \\
2006\end{array}$ & $\begin{array}{c}\text { № } \\
\text { BVBB }\end{array}$ & $\begin{array}{l}\text { POB. } \\
\text { Vuln. }\end{array}$ & $\begin{array}{l}\% \text { POB. } \\
\text { Vuln. }\end{array}$ \\
\hline Alcoy & 65.514 & 2 & 13.877 & 21,18 & 58.358 & 3 & 16.245 & 27,84 & 60.590 & 0 & 0 & - \\
\hline Alicante & 265.473 & 4 & 23.788 & 8,96 & 284.580 & 6 & 39.220 & 13,78 & 322.431 & 0 & 0 & - \\
\hline Benidorm & 42.371 & - & - & - & 51.873 & 1 & 5.168 & 9,96 & 67.627 & 2 & 11.325 & 16,75 \\
\hline Elche & 188.062 & 8 & 84.877 & 45,13 & 194.767 & 10 & 74.752 & 38,38 & 219.032 & 1 & 5.583 & 2,55 \\
\hline Elda & 54.350 & 5 & 43.588 & 80,2 & 51.593 & 3 & 16.506 & 31,99 & 55.135 & 0 & 0 & - \\
\hline Orihuela & 49.038 & - & - & - & 54.390 & 1 & 4.587 & 8,43 & 77.979 & 2 & 9.188 & 11,78 \\
\hline Torrevieja & 24.839 & - & - & - & 50.953 & 2 & 7.783 & 15,27 & 92.034 & 9 & 65.785 & 71,48 \\
\hline $\begin{array}{l}\text { Castelló } \\
\text { de la Plana }\end{array}$ & 134.213 & - & 0 & 0 & 147.667 & 1 & 4.625 & 3,13 & 172.110 & 7 & 44.445 & 25,82 \\
\hline Gandía & 51.803 & 1 & 3.983 & 7,69 & 59.850 & 0 & 0 & - & 74.827 & 4 & 26.827 & 35,85 \\
\hline Paterna & 40.855 & - & - & - & 46.974 & - & - & - & 57.343 & 0 & & - \\
\hline Sagunt & 55.457 & 0 & 0 & - & 56.471 & 0 & 0 & - & 62.702 & 0 & 0 & - \\
\hline Torrent & 56.564 & 2 & 9.431 & 16,67 & 65.417 & 3 & 22.035 & 33,68 & 74.616 & 0 & 0 & - \\
\hline Valencia & 752.909 & 2 & 19.587 & 2,6 & 738.441 & 8 & 53.524 & 7,25 & 805.304 & 13 & 88.673 & 11,01 \\
\hline Total & - & 24 & 199.131 & - & - & 38 & 244.445 & - & - & 38 & 251.826 & - \\
\hline
\end{tabular}

Fuente: Elaboración propia a partir del Atlas de Barrios Vulnerables (2015).

Sin embargo, desde el ámbito legislativo cabe puntualizar que con el Decreto 157/1988, de 11 de octubre, del Consell de la Generalitat Valenciana, por el que se establece el Plan Conjunto de Actuación de Barrios de Acción Preferente ${ }^{7}$, se destaca la existencia de barrios vulnerables en municipios como Paterna, Alicante o Burjassot que el Atlas de Barrios Vulnerables no contempla. En este sentido, desde el nivel autonómico la Conselleria de Vivienda, Obras Públicas y Urbanismo ha desarrollado el VEUS al objeto de cartografiar la vulnerabilidad urbana en la Comunitat Valenciana a escala de sección censal (3.470 recintos de análisis) y con las siguientes variables:

\footnotetext{
${ }^{5}$ Para el año 1991, inicialmente fueron analizadas un total de 9 ciudades mientras que en el año 2011 se aumentó dicha cifra hasta un total de 12 ciudades.

${ }^{6}$ Los datos de 2006, fueron publicados en el año 2015.

${ }^{7}$ De acuerdo con la normativa, son Barrios de Acción Preferente (BAP): El Barrio de "Las 1.000 viviendas" de Alicante, Barrio de "La Tafalera" de Elda, Barrio "Los Palmerales" de Elx, Barrio "San Agustín y San Marcos" de Castelló de la Plana, Barrio "613 viviendas" de Burjassot, Barrio "San José" de Xirivella, Barrio "La Coma" de Paterna, Barrio "Baladre" de Sagunto y Barrio "Zorrilla" de Torrent. No obstante, el Barrio de Zorrilla (Torrent) y el de "San José" fueron suprimidos del decreto tras su demolición en 1993. Asimismo, cabe destacar que en los años noventa, se incorporó el Barrio del Cristo (Aldaia-Quart) a la categoría de BAP (Caravantes y Serrano, 2016).
} 


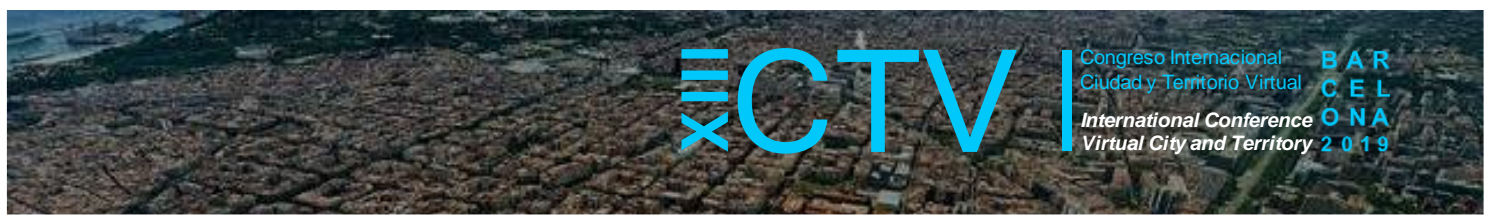

Tabla 3. Variables de análisis del VEUS

\begin{tabular}{|c|l|}
\hline CATEGORÍA DE LA VARIABLE & VARIABLES \\
\hline \multirow{4}{*}{ RESIDENCIAL } & Superficie media por habitante de los inmuebles residenciales \\
\cline { 2 - 2 } & Valor catastral \\
\cline { 2 - 2 } & Accesibilidad \\
\hline \multirow{3}{*}{ SOCIOECONÓMICA } & Población sin estudios \\
\cline { 2 - 2 } & Tasa de desempleo registrado estimado con perspectiva de género \\
\cline { 2 - 2 } & Riesgo de pobreza y/o exclusión social \\
\hline \multirow{3}{*}{ SOCIODEMOGRÁFICA } & Población vulnerable con perspectiva de género \\
\cline { 2 - 2 } & Hogares vulnerables con perspectiva de género \\
\cline { 2 - 2 } & Población inmigrante con perspectiva de género \\
\hline
\end{tabular}

Fuente: Elaboración propia a partir de VEUS (2018).

Como resultado de ello, se identifican tres tipos de vulnerabilidad:

1) vulnerabilidad integral (secciones censales en las que tres dimensiones de vulnerabilidad están presentes con niveles altos);

2) polivulnerabilidad (el factor común de estas secciones censales es que al menos dos de las dimensiones tienen valores altos o hay dos dimensiones medias y una alta);

3) vulnerabilidad residual (secciones censales con el resto de combinaciones posibles).

En función de estas tipologías se han identificado 256 secciones censales con vulnerabilidad integral, 1160 con polivulnerabilidad y 2054 con vulnerabilidad residual. La misma herramienta de análisis, permite identificar los Espacios Urbanos Sensibles (EUS) entendidos como "aquellas zonas urbanas delimitadas sobre la base de las diversas dimensiones de vulnerabilidad: vulnerabilidad físico-residencial, vulnerabilidad socioeconómica y vulnerabilidad sociodemográfica" (VEUS, 2018, pág. 15). Esta delimitación, abarca un mayor espacio de análisis que el ofrecido desde el Ministerio de Fomento, dado que va más allá de la mera delimitación administrativa de 'barrio'. En este caso, la identificación de los EUS en la Comunitat Valenciana se integran en un total de 830 secciones censales de los 3.470 recintos de análisis, es decir, los EUS tienen una representación de un 24\% englobando a 1.531.261 personas $\left(31 \%\right.$ sobre la población total de la CV). ${ }^{8}$

Tabla 4. Espacios Urbanos Sensibles en la Comunitat Valenciana

\begin{tabular}{|c|c|c|c|}
\hline PROVINCIA & $\begin{array}{c}\text { NNo. de Secciones } \\
\text { Censales analizadas }\end{array}$ & $\begin{array}{c}\text { № de Secciones } \\
\text { Censales en EUS }\end{array}$ & $\begin{array}{c}\text { \% de EUS con respecto al } \mathbf{n}^{\circ} \\
\text { de secciones censales }\end{array}$ \\
\hline Alicante & 1.222 & 458 & $37,17 \%$ \\
\hline Castellón & 458 & 48 & $10,48 \%$ \\
\hline València & 1.808 & 324 & $17,92 \%$ \\
\hline TOTAL & $\mathbf{3 . 4 7 0}$ & $\mathbf{8 3 0}$ & $\mathbf{2 3 , 9 1}$ \\
\hline
\end{tabular}

Fuente: Elaboración propia a partir de VEUS (2018)

De acuerdo con estos datos y como puede apreciarse en el Mapa 2, la provincia de Alicante es la que mayor territorio con EUS concentra, tanto en la zona del litoral levantino como en la zona del interior marcando una destacable concentración de vulnerabilidad urbana en la zona sur de la provincia.

\footnotetext{
${ }^{8}$ A fecha de 1 de enero de 2016 la población de la Comunitat Valenciana ascendía a un total de 4.959 .968 personas.
} 


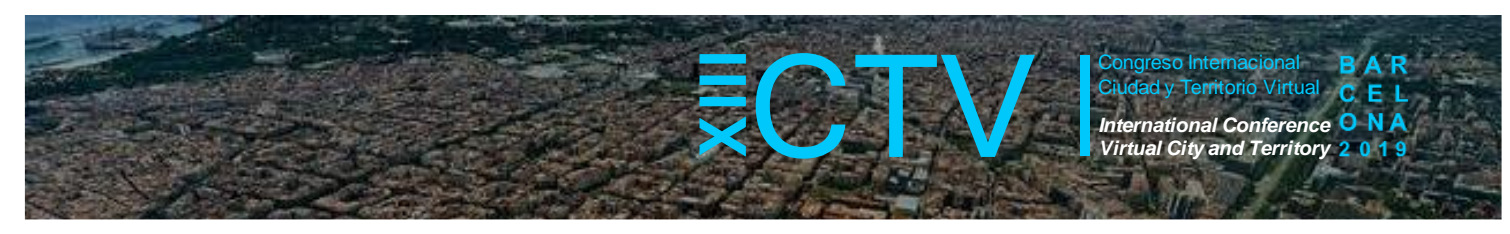

\section{Mapa 2. Espacios Urbanos Sensibles y análisis de la vulnerabilidad en la Comunitat Valenciana}

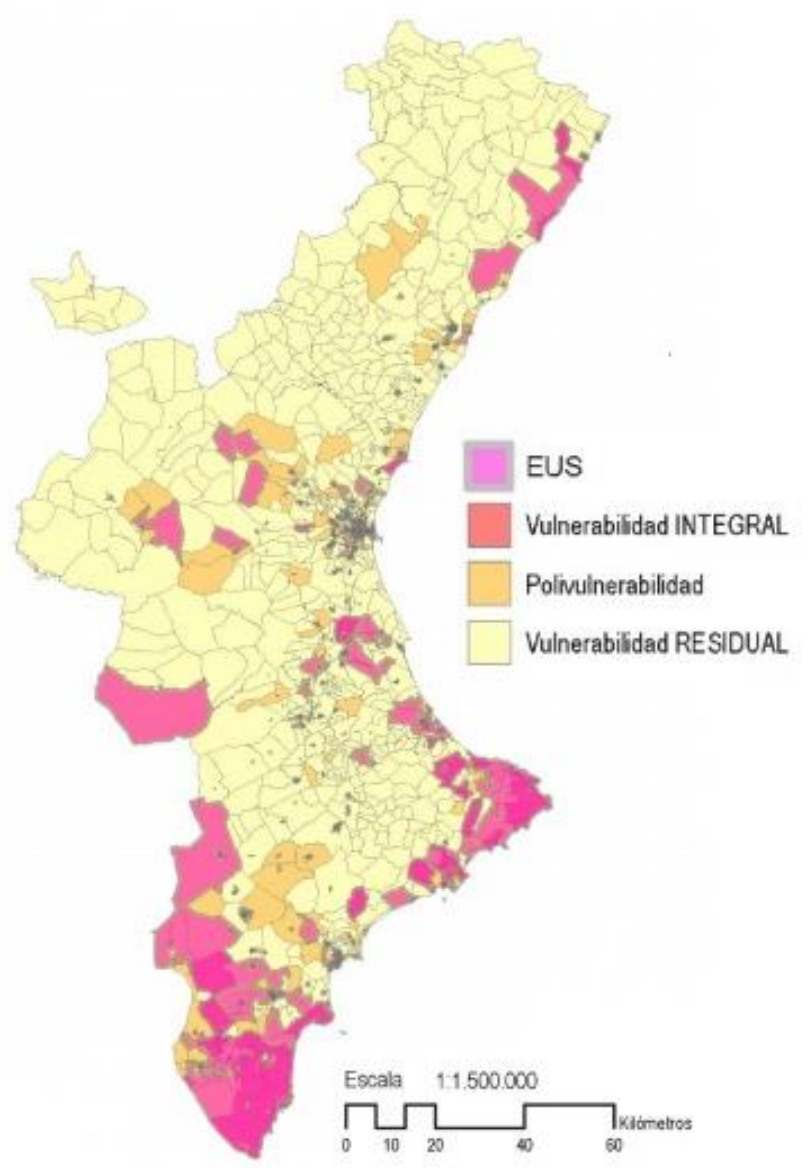

Fuente: VEUS (2018).

Además de ello, si contemplamos la cartografía desde la variable residencial, en el Mapa 3 aparece reflejado en color rojo la alta vulnerabilidad residencial. En el caso de Alicante, esta variable es muy significativa en lo relativo a los Espacios Urbanos Sensibles dado, que coincide territorialmente con la ubicación de los mismos en la zona del litoral y del sur provincial. Del mismo sucede en la provincia de València y de Castellón, donde las zonas en las que se identifican EUS presentan unos altos niveles de vulnerabilidad residencial.

Tal y como fue destacado en epígrafes anteriores, la vulnerabilidad y la exclusión residencial es una variable determinante en el estudio de los barrios vulnerables. Además de la variable residencial, resulta significativo destacar la variable demográfica en lo concerniente a la concentración de población con características similares como consecuencia de "una serie de inercias que forman parte del sistema en que vivimos, que se encuentran presentes tanto en las políticas que conducen a la pobreza como, en ocasiones, en las fuerzas que intentan combatirlas (Varea, 2016, pág. 100).

En el Mapa 2, se aprecia este tipo de concentración, en el que la Comunitat Valenciana se encuentra entre aquellas CCAA con mayores índices de población gitana e inmigrante, junto Andalucía, Madrid y Cataluña. 
Mapa 3. Vulnerabilidad residencial en la Comunitat Valenciana

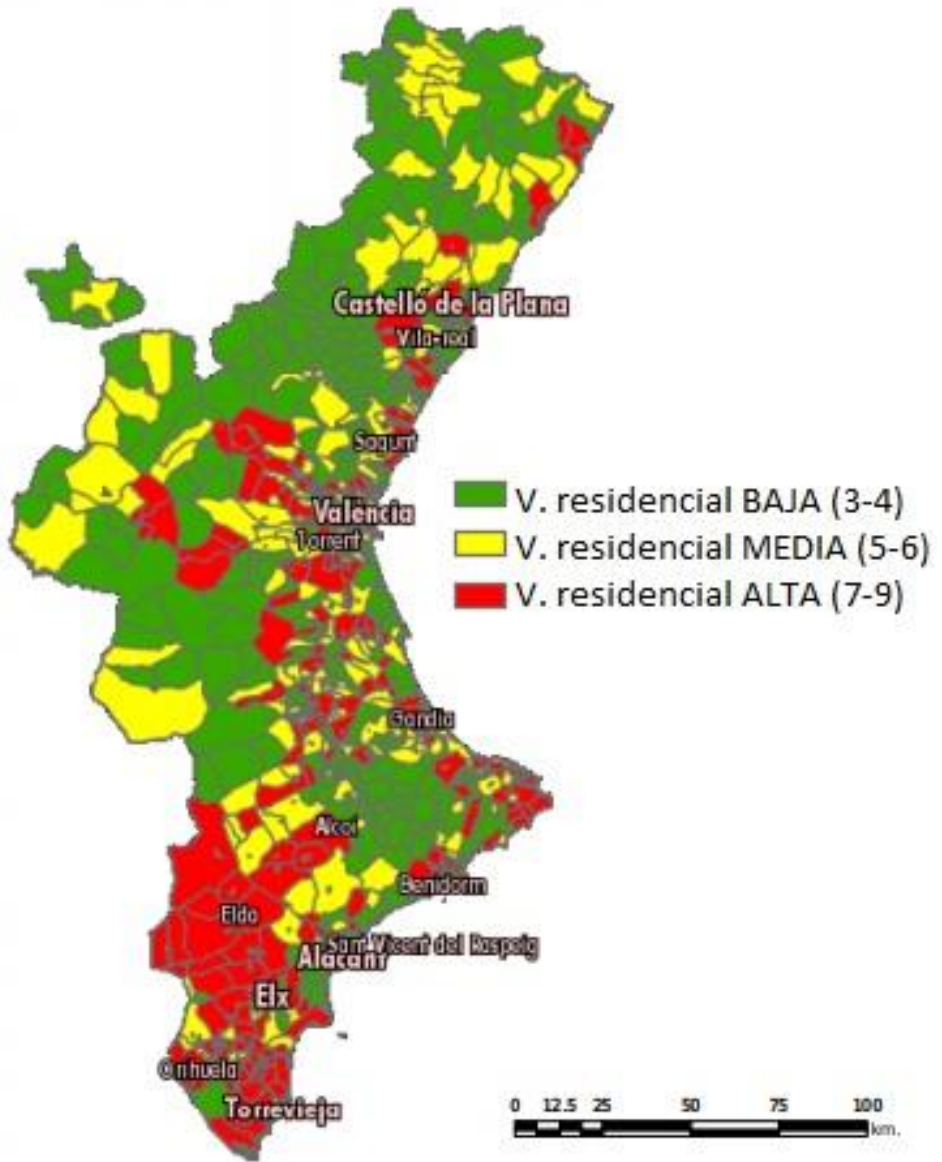

Fuente: Elaboración propia a partir de VEUS (2018).

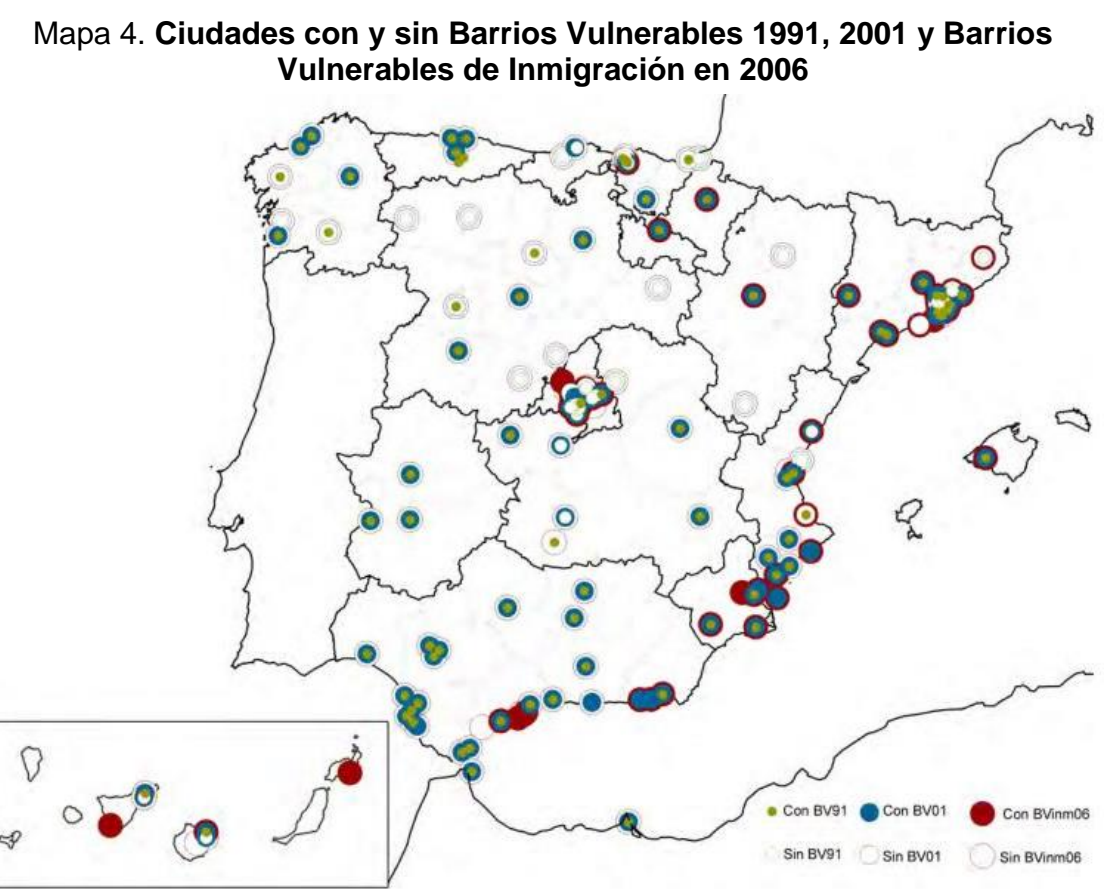

Fuente: Atlas de Barrios Vulnerables (2015, pág. 15) 


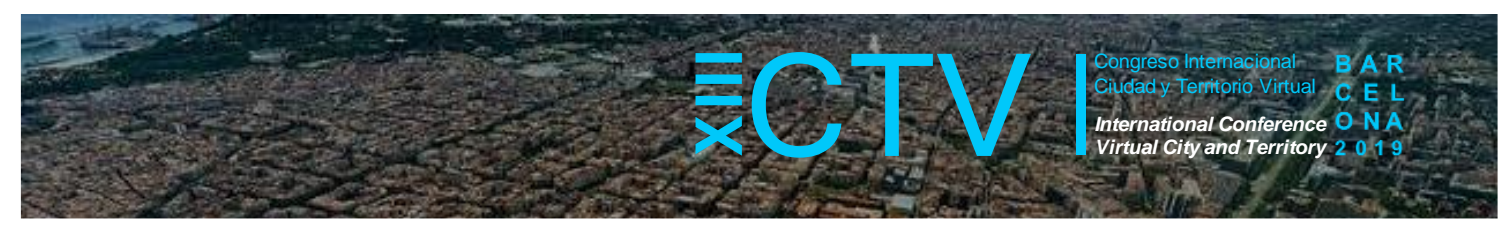

Se han experimentado importantes avances en materia residencial "en la actualidad, las viviendas en condiciones muy precarias (viviendas de transición, deterioradas, móviles y chabolas) tan sólo suponen el $4,78 \%$ de las viviendas de las familias gitanas. No obstante, este porcentaje, además de ser ligeramente superior al registrado en 2007, corresponde a un incremento de unas 100 viviendas en condiciones precarias, puesto que el número de infraviviendas asciende a 592 (493 en 2007)" (Ministerio de Fomento, 2018, pág. 300).

A pesar de ello, el porcentaje de población gitana residente en las zonas periféricas de las ciudades se ha mantenido estable puesto que "continúan existiendo los 11 asentamientos segregados detectados en 2007, que suponen el 3,59\% del total en 2015 y el 3,13\% del total en 2007, mientras que en 1991 representaban el 24\% del total" (Ministerio de Fomento, 2018, pág. 301 - 302). Si consideramos los datos respecto a las tendencias de población gitana en barrios o asentamientos de la Comunitat Valenciana, como aparece reflejado en la Tabla 3, el número de estos se encuentra en crecimiento o estacionamiento:

Tabla 3. Tendencias de la población gitana en barrios o asentamientos de la Comunitat Valenciana

\begin{tabular}{|c|c|c|c|c|c|c|c|c|c|c|c|c|c|c|}
\hline & \multicolumn{4}{|c|}{ VALĖNCIA } & \multicolumn{4}{|c|}{ ALICANTE } & \multicolumn{4}{|c|}{ Castellón } & \multirow{3}{*}{ 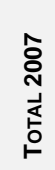 } & \multirow{3}{*}{ 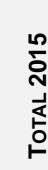 } \\
\hline & \multicolumn{2}{|c|}{2007} & \multicolumn{2}{|c|}{2015} & \multicolumn{2}{|c|}{2007} & \multicolumn{2}{|c|}{2015} & \multicolumn{2}{|c|}{2007} & \multicolumn{2}{|c|}{2015} & & \\
\hline & $\begin{array}{l}\text { №BB/ } \\
\text { Asent }\end{array}$ & $\%$ & $\begin{array}{l}\text { №BB/ } \\
\text { Asent }\end{array}$ & $\%$ & $\begin{array}{l}\text { №BB/ } \\
\text { Asent }\end{array}$ & $\%$ & $\begin{array}{l}\text { №BB/ } \\
\text { Asent }\end{array}$ & $\%$ & $\begin{array}{l}\text { №BB/ } \\
\text { Asent }\end{array}$ & $\%$ & $\begin{array}{l}\text { №BB/ } \\
\text { Asent }\end{array}$ & $\%$ & & \\
\hline $\begin{array}{c}\text { En } \\
\text { crecimiento }\end{array}$ & 51 & 30 & 51 & 30,7 & 47 & 32,2 & 39 & 38,2 & 3 & 6,8 & 11 & 30,6 & 101 & 101 \\
\hline Estacionado & 86 & 50,6 & 91 & 54,8 & 83 & 56,9 & 58 & 56,9 & 37 & 84,1 & 25 & 69,4 & 206 & 173 \\
\hline $\begin{array}{c}\text { En } \\
\text { reducción }\end{array}$ & 13 & 7,6 & 24 & 14,5 & 11 & 7,5 & 5 & 4,9 & 2 & 4,5 & 0 & 0 & 26 & 29 \\
\hline $\begin{array}{l}\text { Tendencia a } \\
\text { desaparecer }\end{array}$ & 11 & 6,5 & 0 & 0 & 1 & 0,7 & 0 & 0 & 2 & 4,5 & 0 & 0 & 14 & 1 \\
\hline $\mathrm{NS} / \mathrm{NC}$ & 9 & 5,3 & 1 & - & 4 & 2,7 & 1 & - & - & - & - & - & 13 & 2 \\
\hline Total & 170 & 100 & 167 & - & 146 & 100 & 103 & & 44 & 100 & 36 & & 360 & 306 \\
\hline
\end{tabular}

Fuente: Elaboración propia a partir de Mapa sobre Vivienda y Comunidad Gitana en España 2007 y 2015.

En las tres provincias objeto de estudio el número de barrios o asentamientos se ha reducido notablemente, aunque el grueso de éstos se encuentra en crecimiento o estacionados, tanto si consideramos el año 2007 como el año 2015. Además, del mismo modo que en el año 2007, la provincia de València continúa englobando el mayor número de barrios o asentamientos con población gitana (167 respecto a los 170 del pasado informe), seguido de Alicante (103 respecto a los 146) y Castellón (306 respecto a los 360 anteriores).

\section{Conclusiones}

Por todo lo expuesto previamente, cabe destacar que tanto la mapificación como la identificación de variables para el reconocimiento de potenciales barrios vulnerables en las Comunidades Autónomas, supone al mismo tiempo el reconocimiento institucional de la existencia de este tipo de entornos, generalmente, ubicados en las periferias de las ciudades donde se engloban pluralidad de factores de vulnerabilidad urbana. 


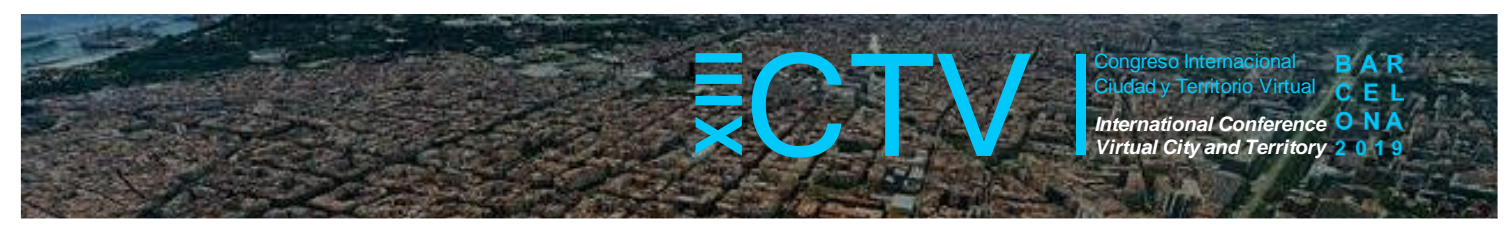

La emergencia de barrios vulnerables en la Comunitat Valenciana no es un fenómeno ex novo sino que tiene su razón de ser en diversidad de causas y dimensiones (residencial, urbanística, social y cultural, económica, educativa, geográfica, institucional...) propias de los procesos de vulnerabilidad y exclusión social.

No obstante, tal y como refieren las distintas fuentes estadísticas de referencia la permanencia de este tipo de enclaves territoriales obliga a repensar por un lado el concepto del derecho a la ciudad en este tipo de entornos, y, por otro lado, las actuaciones institucionales desarrolladas desde los poderes públicos. Cabría preguntarse pues, ¿existe el derecho a la ciudad en los espacios vulnerables o es una asignatura pendiente para los poderes públicos?

Tanto el derecho a la ciudad como el derecho a la vivienda, implican mutuamente la garantía de gozar de una serie de derechos de ciudadanía con base territorial. En este sentido, no puede entenderse el derecho a la ciudad sin la garantía del derecho a la vivienda, el derecho a la educación, el derecho a la sanidad o el derecho a los servicios sociales. En la vida urbana de los barrios vulnerables, la concatenación de factores de riesgo (trabajo inestable, escasez de recursos...) dificultan no únicamente su tenencia (en el caso de la vivienda), sino también el acceso y el mantenimiento a la misma.

Por su parte, tanto la identificación como el reconocimiento (legislativo en el caso de la Comunitat Valenciana) de barrios vulnerables, supone el primer paso para abordar los fenómenos de la vulnerabilidad y de la exclusión social en estos entornos. La inclusión en las agendas políticas de los gobiernos (nacionales, regionales y locales) en el abordaje de este tipo de cuestiones, constituye el segundo paso al objeto de hacer efectivo el derecho a la ciudad. No obstante, los poderes públicos no pueden seguir escudándose en la ya consolidada crisis económica, encontrando resistencias y argumentos opuestos a desarrollar políticas públicas eficaces en este tipo de barrios.

Además de ello, la gobernanza territorial, ha de partir de la potenciación de factores de protección endógenos y exógenos existentes en las ciudades para hacer frente a los factores de riesgo. La participación ciudadana en la fase de diagnóstico, implementación y evaluación, y la promoción de la cohesión social en pro del desarrollo local suponen los pilares fundamentales para la elaboración de una política pública orientada a repensar el potencial territorial y su vinculación con los grupos vulnerables.

Agradecimientos: Agradecer al Ministerio de Ciencia, Innovación y Universidades la financiación de la Tesis Doctoral titulada: "Ciudades sostenibles: el análisis de la vulnerabilidad urbana. Propuesta de una política urbana de intervención en espacios vulnerables de la Comunitat Valenciana" cuyos primeros avances son presentados en esta ponencia.

Conflicto de Intereses: La autora declara que no hay conflicto de intereses.

\section{Bibliografía}

Busquet, G., y Garnier, J. P. (2011). Un pensamiento urbano todavía contemporáneo. Las vicisitudes de la herencia lefebvriana. Urban, (2), pp. 41-57. 


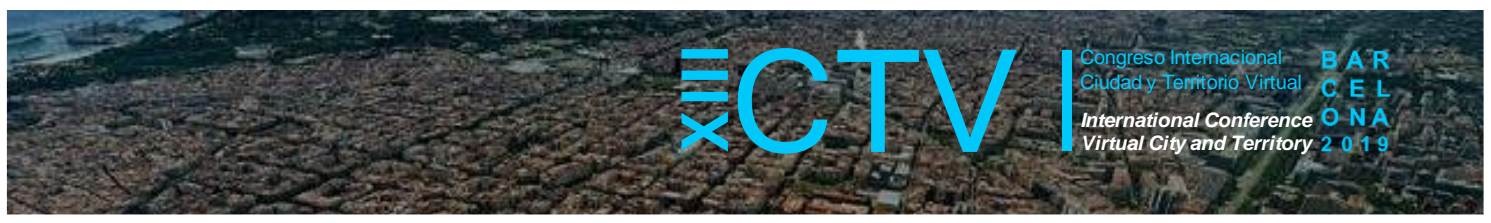

Caravantes, G. M. (2018). El derecho a la ciudad y el derecho a la vivienda: el análisis del ODS 11 desde la exclusión residencial en el Sur y en el Norte. En Vicente Bellver (coord.). II Congreso Internacional sobre Derechos Humanos: Los Derechos Humanos y los Objetivos de Desarrollo Sostenible (pp. 103-121). València, España: Fundación Mainel.

Caravantes, G. M., y Serrano, A. I. (2016). Los Servicios Sociales para la exclusión social: Barrios de Acción Preferente. En L. Martínez-Martínez, et al., (eds.). Los Servicios Sociales en la provincia de Valencia: análisis territorial, estado de la cuestión. (pp. PUV: IIDL. Colección Desarrollo Territorial.

Conselleria d'Habitatge, Obres Públiques i Urbanisme (2018). Visor d'Espais Urbans Sensibles. Recuperado de https://bit.ly/2KJ9QDP

Decreto 157/1988, de 11 de octubre, del Consell de la Generalitat Valenciana, por el que se establece el Plan Conjunto de Actuación de Barrios de Acción Preferente

Fundación FOESSA. (2014). VII Informe sobre exclusión y desarrollo social en España. Recuperado de: http://www.foessa2014.es/informe/

Fundación FOESSA. (2013). Encuestas sobre Integración Social y Necesidades Sociales (EINSFOESSA).

Fundación FOESSA. (2008). La exclusión social en España, un espacio diverso y disperso en intensa transformación. Recuperado de: https://bit.ly/31JGdJI

Hernández-Aja, A., Matesanz, Á. y García-Madruga, C. (2015). Atlas de Barrios Vulnerables de España. Instituto Juan de Herrera (IJH): Madrid.

Portal Estadístico de la Generalitat Valenciana (2019). Encuesta de Condiciones de Vida. Recuperado de https://bit.ly/31NxUfU

Portal Estadístico de la Generalitat Valenciana (2017). Encuesta de Condiciones de Vida. Recuperado de http://bit.ly/2tvasll

Jacobs, J. (2011). Muerte y vida de las grandes ciudades. Capitán Swing: Madrid.

Lefebvre, H. (1969). El derecho a la ciudad. Península: Madrid.

Ministerio de Sanidad, Igualdad y Servicios Sociales (2018). Mapa sobre Vivienda y Comunidad Gitana en España. Anexo I: Información a nivel autonómico y provincial. Recuperado de https://bit.ly/2MoXV1g

Ministerio de Sanidad, Igualdad y Servicios Sociales (2007). Mapa sobre Vivienda y Comunidad Gitana en España: Madrid.

ONU - HÁBITAT (2015). UN-Habitat Global Activities Report. Increasing Synergy for Greater National Ownership. Recuperado de https://bit.ly/2KFrE3W 


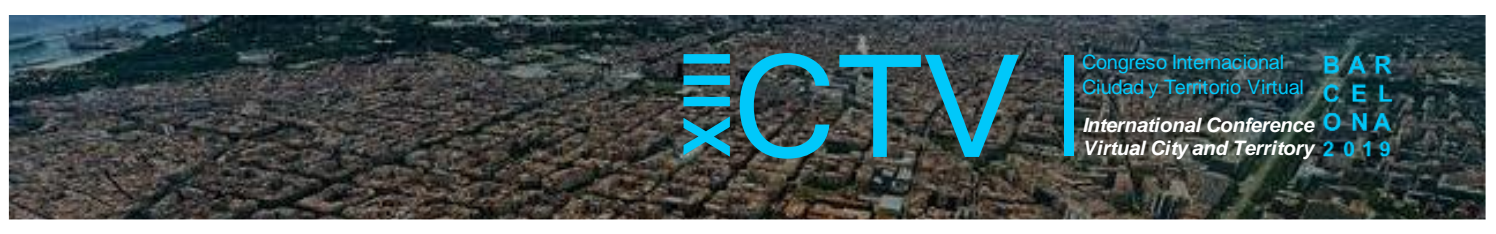

Subirats, J. (DIR.) et al. (2004). Pobreza y exclusión social. Un análisis de la realidad española y europea. Barcelona: Fundación "La Caixa", Recuperado de http://bit.ly/1MgakdD

Uceda-Maza, F.X. et al., (2018). Bienestar y políticas sociales, en Ariño, A. (dir.) y García-Pilán, P. (coord.). La sociedad valenciana en transformación (1975-2025). València: PUV. Disponible en https://bit.ly/2ULwnU7

Uceda-Maza, F. X. (2017). Una agenda valenciana de transformació social: un Nou Model Social Valencià. Revista Valenciana d'Estudis Autonòmics. 62, pp. 89 - 120.

Varea, A., et al. (2016): Construir la ciudad colectivamente. De la exclusión social al bien común. En: E. Lluch, et al. (eds.). Medio Ambiente y Política Social, Análisis y Perspectivas Comunitat Valenciana 2016 (pp. 99-112). Observatorio de Investigación sobre Pobreza y Exclusión en la Comunitat Valenciana. 